\title{
Effects of a Prenatal Lifestyle Intervention in Routine Care on Maternal Health Behaviour in the First Year Postpartum-Secondary Findings of the Cluster-Randomised GeliS Trial
}

\author{
Kristina Geyer ${ }^{1,+} \mathbb{D}$, Monika Spies ${ }^{1,+} \mathbb{D}$, Julia Günther ${ }^{1}$, Julia Hoffmann ${ }^{1} \mathbb{D}$, Roxana Raab ${ }^{1} \mathbb{D}$, Dorothy Meyer ${ }^{1} \mathbb{D}$, \\ Kathrin Rauh ${ }^{1,2}$ and Hans Hauner $1, *$ (D) \\ 1 Else Kröner-Fresenius-Centre for Nutritional Medicine, Institute of Nutritional Medicine, School of Medicine, \\ Technical University of Munich, Georg-Brauchle-Ring 62, 80992 Munich, Germany; k.geyer@tum.de (K.G.); \\ monika.spies@tum.de (M.S.); julia.guenther@tum.de (J.G.); julia.hoffmann@tum.de (J.H.); \\ roxana.raab@tum.de (R.R.); dora.meyer@tum.de (D.M.); kathrin.rauh@tum.de (K.R.) \\ 2 Competence Centre for Nutrition (KErn), Am Gereuth 4, 85354 Freising, Germany \\ * Correspondence: hans.hauner@tum.de; Tel.: +49-(0)89-289249-21 \\ + These authors contributed equally to this work and share first authorship.
}

Citation: Geyer, K.; Spies, M.; Günther, J.; Hoffmann, J.; Raab, R.; Meyer, D.; Rauh, K.; Hauner, H. Effects of a Prenatal Lifestyle Intervention in Routine Care on Maternal Health Behaviour in the First Year Postpartum-Secondary Findings of the Cluster-Randomised GeliS Trial. Nutrients 2021, 13, 1310. https://doi.org/10.3390/nu13041310

Academic Editors: Judith Rankin, Nicola Heslehurst and Laura Gaudet

Received: 18 March 2021

Accepted: 13 April 2021

Published: 15 April 2021

Publisher's Note: MDPI stays neutral with regard to jurisdictional claims in published maps and institutional affiliations.

Copyright: (c) 2021 by the authors. Licensee MDPI, Basel, Switzerland. This article is an open access article distributed under the terms and conditions of the Creative Commons Attribution (CC BY) license (https:/ / creativecommons.org/licenses/by/ $4.0 /)$.

\begin{abstract}
Lifestyle interventions during pregnancy were shown to beneficially influence maternal dietary behaviour and physical activity, but their effect on health behaviour after delivery is unclear. The objective of this secondary analysis was to investigate the sustained effect of a lifestyle intervention in routine care on maternal health behaviour during the first year postpartum. The cluster-randomised controlled "Healthy living in pregnancy" (GeliS) study included 2286 pregnant women. Data on maternal health behaviour were collected at 6-8 weeks (T1pp) and one year postpartum (T2pp) using validated questionnaires. The intervention group showed a lower mean intake of fast food (T1pp: $p=0.016$; T2pp: $p<0.001$ ) and soft drinks (T1pp: $p<0.001$ ), a higher mean intake of vegetables (T2pp: $p=0.015$ ) and was more likely to use healthy oils for meal preparation than the control group. Dietary quality rated by a healthy eating index was higher in the intervention group (T1pp: $p=0.093$; T2pp: $p=0.043$ ). There were minor trends towards an intervention effect on physical activity behaviour. The proportion of smokers was lower in the intervention group $(p<0.001$, both time points). The lifestyle intervention within routine care modestly improved maternal postpartum dietary and smoking behaviours.
\end{abstract}

Keywords: postnatal; postpartum lifestyle; maternal diet; dietary behaviour; physical activity; smoking; obesity prevention; lifestyle intervention; primary care

\section{Introduction}

Healthy lifestyle habits during the time of pregnancy and postpartum are important for optimising maternal and child health in the short- and long-term [1,2]. The modern Western lifestyle, with a high-caloric diet, low physical activity (PA) and high risk of weight gain may be detrimental for pregnancy and the early postpartum period [2,3]. In Germany, 67\% of pregnant women gain weight in excess of the recommendations set forth by the Institute of Medicine (IOM) [4,5], increasing the likelihood of postpartum weight retention [6-8], weight gain in subsequent pregnancies [9] and overweight later in life [10]. Growing evidence suggests that a healthy lifestyle during pregnancy, but also after delivery, is associated with multiple benefits such as with an adequate body mass index (BMI) [11-13], cardio-metabolic benefits [14-16], aerobic fitness, insulin sensitivity and improvements in overall psychological wellbeing [15,17-19]. In order to support women during pregnancy and the postpartum period, recommendations on optimal dietary behaviour and regular PA have been published [20-23]. 
There is evidence that antenatal lifestyle interventions are able to improve maternal diet and PA during gestation [24,25] and achieve a reduction in excessive gestational weight gain (GWG) [26]. However, adhering to a healthy lifestyle during the postpartum period has been shown to be challenging, and several studies indicate a decrease in dietary quality and PA after delivery [27-31]. This might be attributed to factors, such as lack of time and sleep, parenting duties and prioritising competing responsibilities over health, which prevent women from implementing or maintaining a healthy diet and PA level [32]. Therefore, supporting women in achieving a healthy lifestyle already in pregnancy might help to sustain healthy routines after delivery. While the effects of lifestyle interventions on maternal diet and PA during pregnancy have been extensively studied [24-26], lasting intervention effects on maternal health behaviour in the postpartum period are poorly explored [33-36]. Moreover, interventions were primarily conducted under fairly controlled conditions in academic settings [26,37] rather than in a primary care setting. To address this research gap, this secondary analysis of the large-scale, cluster-randomised "Gesund leben in der Schwangerschaft" (GeliS; "Healthy living in pregnancy") study investigated if a comprehensive lifestyle intervention, embedded in routine care, was able to influence maternal lifestyle during the first year postpartum.

\section{Materials and Methods}

\subsection{The GeliS Study: Design and Setting}

The GeliS study is a prospective, multicentre, cluster-randomised, controlled, open intervention trial that took place in five administrative regions in Bavaria, Germany. One control and one intervention district per region were matched according to birth figures, sociodemographic and geographic criteria via a pairwise cluster-randomisation. The study was conducted in participating gynaecological and midwifery practices within the 10 districts. More information on the study design and setting is available in the published study protocol [38]. In total, 2286 pregnant women were recruited in the GeliS trial [39]. The primary goal of the GeliS study was to reduce the proportion of women with excessive GWG according to the IOM criteria [5] through a comprehensive lifestyle intervention programme alongside routine care visits. Results on primary and secondary endpoints have already been published [8,39-45]. This secondary analysis focuses on the effect of the GeliS lifestyle intervention programme on maternal health behaviour during the first 12 months postpartum. For this purpose, we investigated differences in health-related lifestyle behaviours, including diet, PA and smoking, between the intervention (IG) and control groups (CG). Additionally, we examined the intervention effect among several subgroups. The study complied with local regulatory requirements and laws and the declaration of Helsinki. The Technical University of Munich Ethics Committee approved the study protocol. The study was registered in the ClinicalTrials.gov Protocol Registration System (NCT01958307).

\subsection{Participants}

Recruitment was carried out by medical personnel between 2013 and 2015 at 71 gynaecological and midwifery practices. Pregnant women who met the following inclusion criteria were considered for the study: Pre-pregnancy BMI $\geq 18.5 \mathrm{~kg} / \mathrm{m}^{2}$ and $\leq 40.0 \mathrm{~kg} / \mathrm{m}^{2}$, singleton pregnancy, age between 18 and 43 years, sufficient German language skills, stage of pregnancy before the 12th week of gestation and provision of written informed consent. Severe complications that interfered with the intervention were reasons for exclusion [38]. During the follow-up period, women were considered to be drop-outs if they could no longer be reached, did not offer contact information or declined further participation [8].

\subsection{Lifestyle Intervention}

The lifestyle intervention programme comprised four comprehensive face-to-face counselling sessions (12th-16th, 16th-20th and 30th-34th week of gestation, and 6-8 weeks postpartum) alongside routine care delivered by trained midwives, medical personnel or 
gynaecologists. The counselling focused on an adequate GWG as recommended by the IOM [5]. Women were encouraged to maintain healthy dietary and PA behaviours according to national and international guidelines $[20,23,46]$. They were informed about the principles of a healthy diet, were motivated to engage in at least $150 \mathrm{~min}$ of moderate-intensity activity per week and encouraged to increase their daily activity level. Additionally, women were educated about the importance of breastfeeding and the avoidance of smoking and alcohol intake during pregnancy and lactation. Women in the CG received routine antenatal care, along with a leaflet that provided general recommendations on a healthy lifestyle and breastfeeding. Details on the GeliS lifestyle intervention and counselling content have been published elsewhere [38].

\subsection{Data Collection and Processing}

Data on baseline maternal characteristics were obtained from a screening questionnaire before the 12th week of gestation. Maternal dietary, PA and smoking data were collected via questionnaires in early pregnancy (before the 12th week of gestation, used as baseline) and late pregnancy (after the 29th week of gestation), and at two time points during the first year postpartum (T1pp: 6-8 weeks postpartum; T2pp: One year postpartum). The intervention effect on antenatal dietary and PA behaviours has already been published elsewhere [41,42]. The analyses presented herein focus on the dietary and PA behaviours during the postpartum period as well as smoking behaviour, for which all four time points of data collection were considered.

Dietary variables were collected via a self-administered and validated food frequency questionnaire (FFQ) originally used for the "German Health Interview and Examination Survey for Adults" (DEGS) trial conducted by the Robert Koch Institute, Berlin, Germany [47]. The utilised DEGS-FFQ was slightly altered from the original version and consisted of 54 questions relating to consumption frequency and portion size of food items and dietary behaviour over the previous four weeks. Additionally, four questions targeted food preparation and dietary choices, vegetarianism and the frequency of fresh food preparation. For the calculation of mean daily intakes, food items were grouped into 17 food groups as recommended by the developers of the DEGS-FFQ (personal communication: Dr. G. Mensink, Robert Koch Institute, 2018). In case of over-reporting of food intake or missing amounts of more than 20 out of 54 food items, questionnaires were excluded from the analysis as described previously [42]. Energy, macronutrient and fibre intake were estimated using the German food composition database ("Bundeslebensmittelschlüssel", version 3.02) using the OptiDiet PLUS software (version 6.0, GOE mbH, Linden, Germany). Women under- or over-reporting energy intake were precluded from the statistical analysis of energy and macronutrient intake as described previously [42]. The DEGS-Healthy Eating Index (DEGS-HEI) developed by the Robert Koch Institute [48] was calculated to evaluate the dietary quality based on the DEGS-FFQ. The index has a range from 0 to 100, with values closer to 100 indicating better adherence to the German Nutrition Society recommendations [48].

A slightly modified version of the validated Pregnancy Physical Activity Questionnaire (PPAQ) [49] was used to collect data on duration, frequency and intensity of PA behaviour. Participants reported the time spent in 32 activities over the past four weeks and had the option to name two additional sport activities that were not listed in the PPAQ in two open-ended questions. According to the calculation instructions of the PPAQ [50] respecting the 2011 Compendium of Physical Activity [51], the estimated amount of time spent in each activity per week was calculated and expressed as multiples of resting energy expenditures in metabolic equivalent of task (MET)-hours per week. Thereby, PA could be classified in different categories of total activity, activity types and activity intensities. The over-reporting of PA data was defined according to others [25] and has been previously reported [41]. The threshold of $\geq 7.5 \mathrm{MET}-\mathrm{h} /$ week in sport activities of moderate intensity or greater was used to indicate whether women achieved the activity level set in national and international PA recommendations [23,52]. This procedure was recommended by the 
developers of the PPAQ (personal communication: Prof. L. Chasan-Taber, University of Massachusetts Amherst, 2018) and has been applied previously [41].

Smoking behaviour of women was assessed with the question 'Do you currently smoke?', which was part of the questionnaires.

\subsection{Statistical Analysis}

The power calculation was based on excessive GWG as the primary endpoint and has already been described elsewhere [38]. Women were included in the present analysis if they provided at least either a diet or a PA questionnaire at T1pp or T2pp and were not pregnant at T2pp. These women were likewise considered for the analysis of smoking behaviour. Group differences in diet and PA variables were estimated using linear regression models fit with generalised estimating equations (GEE), as recommended for cluster-randomised trials [53]. The models were adjusted for pre-pregnancy BMI category, age, parity, baseline dietary assessment for dietary variables or baseline PA assessment for PA variables, respectively, and time interval between questionnaire completion date and offspring birth date. Dichotomised variables were compared using binary logistic regression models fit with GEEs and adjusted for the same covariates. The changes in dietary and PA behaviours between T1pp and T2pp (time effects) were investigated using linear mixed models for repeated measures adjusted for pre-pregnancy BMI category, age and parity. The group differences in smoking behaviour were analysed using binary logistic regression models fit with GEEs as described above. For the smoking assessment in early pregnancy, the regression model was adjusted for pre-pregnancy BMI category, age and parity. For late pregnancy, the model was additionally adjusted for baseline smoking assessment. Similar GEE models, as mentioned above, were applied in exploratory subgroup analyses according to maternal age, pre-pregnancy BMI category, educational level and parity. A post-hoc analysis on potential interactions of group assignment with these factors was performed to screen for influencing factors on the treatment effect. Across all analyses, a $p$-value $<0.05$ was considered statistically significant. Data analysis was performed using SPSS software (IBM SPSS Statistics for Windows, version 26.0, IBM Corp, Armonk, NY, USA).

\section{Results}

\subsection{Flow-Chart and Maternal Characteristics}

In total, 2261 participants were recruited and assigned to the IG $(n=1139)$ or the CG $(n=1122)$ (Figure 1). Diet and/or PA data of 1899 women (84.0\%) were collected on either one or both postpartum assessments (IG: $n=952$; CG: $n=947$ ). At $6-8$ weeks postpartum, a total of 1791 women provided valid dietary data (IG: $n=898$; CG: $n=893$ ), 1812 provided valid PA data (IG: $n=907$; CG: $n=905$ ) and 1815 women provided data on smoking behaviour (IG: $n=905$; CG: $n=910$ ). During the first year postpartum, 101 women in the IG and 114 women in the CG were lost to follow-up, and 75 women were excluded from analyses due to subsequent pregnancies. At the end of the first year postpartum, valid dietary data from 1568 women (IG: $n=791$; CG: $n=777$ ), valid PA data from 1551 women (IG: $n=784$; CG: $n=767$ ) and smoking data from 1557 women (IG: $n=785$; CG: $n=772$ ) were available. With regard to all women who remained in the study until T1pp, the drop-out rate over the course of the one-year follow-up was $10.8 \%$. 


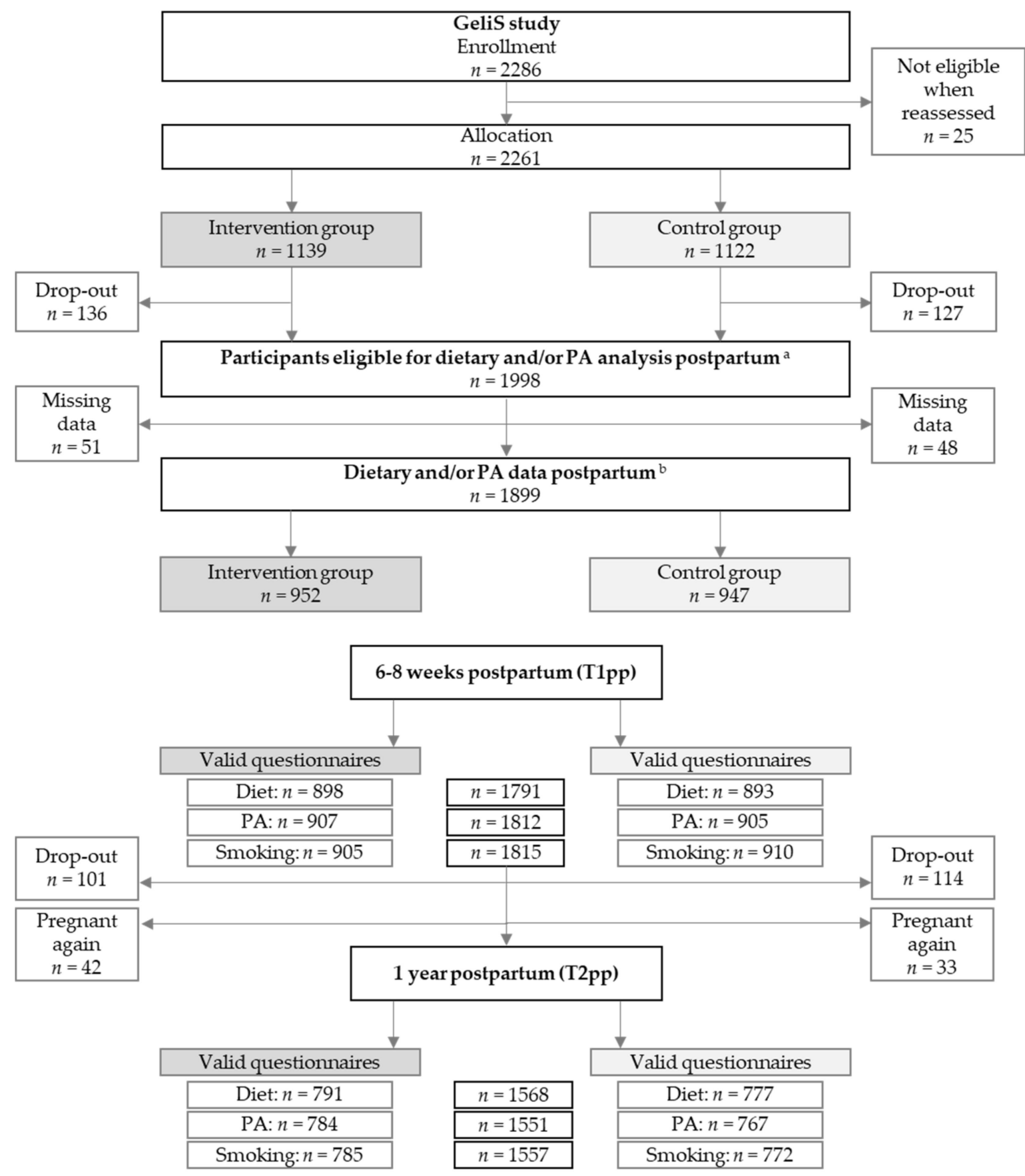

Figure 1. Flow of study participants. Abbreviations: T1pp: Assessment 6-8 weeks after delivery; T2pp: Assessment one year after delivery; PA: Physical activity. ${ }^{a}$ Women who remained in the study until T1pp. ${ }^{b}$ Women who provided PA and/or dietary data at T1pp or T2pp.

As shown in Table 1, mean age, self-reported pre-pregnancy weight, pre-pregnancy BMI and GWG were comparable between the IG and the CG. The majority of women $(65.5 \%)$ had a normal pre-pregnancy weight, while $22.9 \%$ had overweight and $11.6 \%$ had obesity. The proportion of women with excessive GWG was similar in both groups (IG: $n=44.6 \%$; CG: $n=44.9 \%)$. More women were primiparous at the time of inclusion in the IG $(63.3 \%)$ compared to women in the CG $(53.7 \%)$. 
Table 1. Maternal characteristics.

\begin{tabular}{|c|c|c|c|}
\hline & $\begin{array}{l}\text { Intervention Group } \\
\quad(n=952)\end{array}$ & $\begin{array}{l}\text { Control Group } \\
(n=947)\end{array}$ & $\begin{array}{c}\text { Total } \\
(n=1899)\end{array}$ \\
\hline Pre-pregnancy age, years ${ }^{a}$ & $30.3 \pm 4.2$ & $30.6 \pm 4.5$ & $30.4 \pm 4.4$ \\
\hline Pre-pregnancy weight, $\mathrm{kg}^{\mathrm{a}}$ & $68.3 \pm 12.9$ & $67.9 \pm 13.5$ & $68.1 \pm 13.2$ \\
\hline Pre-pregnancy BMI, $\mathrm{kg} / \mathrm{m}^{2} \mathrm{a}$ & $24.4 \pm 4.3$ & $24.3 \pm 4.5$ & $24.3 \pm 4.4$ \\
\hline \multicolumn{4}{|l|}{ Pre-pregnancy BMI category, $n(\%)$} \\
\hline BMI $18.5-24.9 \mathrm{~kg} / \mathrm{m}^{2}$ & $615 / 952(64.6 \%)$ & $628 / 947(66.3 \%)$ & $1243 / 1899(65.5 \%)$ \\
\hline BMI $25.0-29.9 \mathrm{~kg} / \mathrm{m}^{2}$ & $230 / 952(24.2 \%)$ & $205 / 947(21.6 \%)$ & $435 / 1899(22.9 \%)$ \\
\hline BMI $30.0-40.0 \mathrm{~kg} / \mathrm{m}^{2}$ & $107 / 952(11.2 \%)$ & $114 / 947(12.0 \%)$ & $221 / 1899(11.6 \%)$ \\
\hline GWG, $\mathrm{kg}^{\mathrm{a}}$ & $14.0 \pm 5.3$ & $14.0 \pm 5.2$ & $14.0 \pm 5.3$ \\
\hline Excessive GWG, $n(\%){ }^{\mathrm{b}}$ & $423 / 948(44.6 \%)$ & $422 / 940(44.9 \%)$ & $845 / 1888(44.8 \%)$ \\
\hline \multicolumn{4}{|l|}{ Educational level, $n(\%)^{\mathrm{c}}$} \\
\hline General secondary school & $131 / 951(13.8 \%)$ & $152 / 946(16.1 \%)$ & $283 / 1897(14.9 \%)$ \\
\hline Intermediate secondary school & $412 / 951(43.3 \%)$ & $389 / 946(41.1 \%)$ & $801 / 1897(42.2 \%)$ \\
\hline High school & $408 / 951(42.9 \%)$ & $405 / 946(42.8 \%)$ & $813 / 1897(42.9 \%)$ \\
\hline \multicolumn{4}{|l|}{ Country of birth, $n(\%)$} \\
\hline Germany & $923 / 952(97.0 \%)$ & $914 / 945(96.7 \%)$ & $1837 / 1897(96.8 \%)$ \\
\hline Others & $29 / 952(3.0 \%)$ & $31 / 945(3.3 \%)$ & $60 / 1897(3.2 \%)$ \\
\hline Caesarean section, $n(\%)$ & $275 / 951(28.9 \%)$ & $256 / 946(27.1 \%)$ & $531 / 1897(28.0 \%)$ \\
\hline Preterm birth, $n(\%)$ & $62 / 949(6.5 \%)$ & $55 / 947(5.8 \%)$ & $117 / 1896(6.2 \%)$ \\
\hline \multicolumn{4}{|l|}{ Parity } \\
\hline Primiparous, $n(\%)$ & $603 / 952(63.3 \%)$ & $509 / 947(53.7 \%)$ & $1112 / 1899(58.6 \%)$ \\
\hline \multicolumn{4}{|l|}{ Multiparous, $n(\%)$} \\
\hline 1 more child & $291 / 952(30.6 \%)$ & $346 / 947(36.5 \%)$ & $637 / 1899(33.5 \%)$ \\
\hline$\geq 2$ more children & $58 / 952(6.1 \%)$ & $92 / 947(9.7 \%)$ & $150 / 1899(7.9 \%)$ \\
\hline
\end{tabular}

Abbreviations: BMI: Body mass index; GWG: Gestational weight gain; SD: Standard deviation. ${ }^{a}$ Mean \pm SD (all such values). ${ }^{b}$ Excessive GWG as classified by the IOM criteria [5]. ${ }^{\mathrm{c}}$ General secondary school: General school, which is completed through year 9; Intermediate secondary school: Vocational secondary school, which is completed through year 10; High school: Academic high school, which is completed through year 12 or 13.

\subsection{Postpartum Dietary Behaviour}

\subsubsection{Intake of Selected Food Groups}

The mean daily intake for a variety of food groups is shown in Table 2. There was no significant evidence of an intervention effect on the mean intake of caffeinated beverages, fruits, nuts, cheese, meat and meat products, and sweets and snacks at both postpartum time points. At T1pp, women in the IG consumed significantly fewer soft drinks $(p<0.001)$, more dairy products $(p=0.012)$ and more fish $(p<0.001)$ than women in the CG. These between-group differences were no longer significant one year after delivery. At T2pp, women in the IG showed a significantly higher mean daily intake of vegetables compared to women in the CG ( $p=0.015)$. Women in the IG consumed slightly less fast food (T1pp: $p=0.016$; T2pp: $p<0.001)$. The mean consumption of caffeinated beverages increased significantly from $6-8$ weeks postpartum to one year postpartum in both groups $(p<0.001$ respectively), whereas the consumption of sweets and snacks decreased in both groups $(p<0.001$ respectively). 
Table 2. Mean daily intake of selected food groups in the intervention and control groups.

\begin{tabular}{|c|c|c|c|c|c|c|c|c|}
\hline & \multirow{2}{*}{ Time Point } & \multicolumn{2}{|c|}{ Intervention Group } & \multicolumn{2}{|c|}{ Control Group } & \multirow{2}{*}{$n^{\mathrm{a}}$} & \multirow{2}{*}{$\begin{array}{l}\text { Adjusted Effect Size b } \\
(95 \% \mathrm{CI})\end{array}$} & \multirow{2}{*}{$\begin{array}{l}\text { Adjusted } \\
p \text { Value }^{\mathrm{b}}\end{array}$} \\
\hline & & $n$ & Mean $\pm S D$ & $n$ & Mean \pm SD & & & \\
\hline \multirow{2}{*}{$\begin{array}{c}\text { Caffeinated } \\
\text { beverages } \\
\text { (ml/day) }\end{array}$} & T1pp & 897 & $172.17 \pm 197.55$ & 893 & $191.48 \pm 235.54$ & 1673 & $3.17(-23.86,30.20)$ & 0.818 \\
\hline & $\begin{array}{c}\text { T2pp } \\
\text { Time effect }\end{array}$ & 791 & $\begin{array}{c}283.26 \pm 339.91 \\
p<0.001^{c}\end{array}$ & 776 & $\begin{array}{c}286.97 \pm 296.64 \\
p<0.001^{c}\end{array}$ & 1483 & $10.10(-4.74,24.94)$ & 0.182 \\
\hline \multirow{3}{*}{$\begin{array}{l}\text { Soft drinks } \\
\text { (ml/day) }\end{array}$} & T1pp & 897 & $161.49 \pm 301.67$ & 893 & $246.42 \pm 617.64$ & 1674 & $-72.44(-107.00,-37.88)$ & $<0.001$ \\
\hline & T2pp & 791 & $160.57 \pm 458.54$ & 776 & $222.57 \pm 592.33$ & 1484 & $-41.04(-90.10,8.02)$ & 0.101 \\
\hline & Time effect & & $p=0.980^{c}$ & & $p=0.124^{c}$ & & & \\
\hline \multirow{3}{*}{$\begin{array}{l}\text { Vegetables } \\
\text { (g/day) }\end{array}$} & T1pp & 897 & $171.49 \pm 156.65$ & 891 & $157.74 \pm 143.82$ & 1670 & $12.81(-4.21,29.83)$ & 0.140 \\
\hline & $\mathrm{T} 2 \mathrm{pp}$ & 790 & $200.39 \pm 173.66$ & 776 & $180.96 \pm 150.26$ & 1481 & $17.90(3.53,32.27)$ & 0.015 \\
\hline & Time effect & & $p<0.001^{\mathrm{c}}$ & & $p<0.001^{\mathrm{c}}$ & & & \\
\hline \multirow{3}{*}{ Fruit (g/day) } & T1pp & 897 & $206.62 \pm 239.97$ & 890 & $197.06 \pm 194.46$ & 1669 & $9.31(-11.90,30.51)$ & 0.390 \\
\hline & T2pp & 790 & $205.80 \pm 192.72$ & 776 & $194.52 \pm 179.86$ & 1481 & $8.62(-14.11,31.36)$ & 0.457 \\
\hline & Time effect & & $p=0.828^{c}$ & & $p=0.965^{c}$ & & & \\
\hline \multirow{3}{*}{ Nuts (g/day) } & T1pp & 891 & $3.13 \pm 7.65$ & 887 & $3.23 \pm 13.69$ & 1659 & $-0.12(-0.50,0.26)$ & 0.537 \\
\hline & $\mathrm{T} 2 \mathrm{pp}$ & 791 & $2.18 \pm 5.82$ & 773 & $2.03 \pm 6.02$ & 1476 & $0.02(-0.33,0.37)$ & 0.916 \\
\hline & Time effect & & $p<0.001^{c}$ & & $p=0.009^{c}$ & & & \\
\hline \multirow{3}{*}{$\begin{array}{l}\text { Dairy } \\
\text { products } \\
\text { (g/day) }\end{array}$} & T1pp & 898 & $344.12 \pm 308.86$ & 893 & $317.24 \pm 302.89$ & 1675 & $30.95(6.70,55.20)$ & 0.012 \\
\hline & T2pp & 791 & $298.96 \pm 260.15$ & 777 & $304.01 \pm 327.06$ & 1484 & $2.88(-18.56,24.32)$ & 0.792 \\
\hline & Time effect & & $p<0.001^{c}$ & & $p=0.172^{c}$ & & & \\
\hline \multirow{3}{*}{$\begin{array}{l}\text { Cheese } \\
\text { (g/day) }\end{array}$} & T1pp & 890 & $110.56 \pm 116.24$ & 883 & $107.21 \pm 103.85$ & 1652 & $1.39(-13.90,16.68)$ & 0.859 \\
\hline & $\mathrm{T} 2 \mathrm{pp}$ & 788 & $97.15 \pm 93.44$ & 774 & $95.78 \pm 103.77$ & 1473 & $2.53(-4.65,9.72)$ & 0.489 \\
\hline & Time effect & & $p=0.003^{c}$ & & $p=0.005^{c}$ & & & \\
\hline \multirow{3}{*}{ Fish (g/day) } & T1pp & 897 & $17.02 \pm 17.09$ & 891 & $15.43 \pm 19.01$ & 1672 & $1.60(0.81,2.39)$ & $<0.001$ \\
\hline & T2pp & 791 & $17.01 \pm 16.53$ & 775 & $16.46 \pm 16.09$ & 1483 & $0.10(-0.59,0.80)$ & 0.768 \\
\hline & Time effect & & $p=0.971^{c}$ & & $p=0.169^{c}$ & & & \\
\hline \multirow{3}{*}{$\begin{array}{l}\text { Meat and } \\
\text { meat products } \\
(\mathrm{g} / \text { day })\end{array}$} & T1pp & 898 & $99.07 \pm 63.20$ & 893 & $100.64 \pm 67.05$ & 1675 & $-1.72(-4.18,0.73)$ & 0.169 \\
\hline & $\mathrm{T} 2 \mathrm{pp}$ & 791 & $97.19 \pm 55.68$ & 777 & $95.53 \pm 62.00$ & 1484 & $1.17(-5.67,8.01)$ & 0.738 \\
\hline & Time effect & & $p=0.371^{c}$ & & $p=0.040^{c}$ & & & \\
\hline \multirow{3}{*}{$\begin{array}{c}\text { Sweets and } \\
\text { snacks } \\
\text { (g/day) }\end{array}$} & T1pp & 898 & $96.34 \pm 88.48$ & 893 & $96.57 \pm 115.94$ & 1676 & $-0.04(-10.83,10.75)$ & 0.994 \\
\hline & T2pp & 791 & $78.22 \pm 68.28$ & 777 & $76.62 \pm 58.13$ & 1485 & $3.66(-1.58,8.90)$ & 0.171 \\
\hline & Time effect & & $p<0.001^{\mathrm{c}}$ & & $p<0.001^{c}$ & & & \\
\hline \multirow{3}{*}{$\begin{array}{l}\text { Fast food } \\
\text { (g/day) }\end{array}$} & T1pp & 898 & $42.83 \pm 33.80$ & 893 & $43.77 \pm 35.24$ & 1675 & $-2.25(-4.08,-0.42)$ & 0.016 \\
\hline & $\mathrm{T} 2 \mathrm{pp}$ & 791 & $38.29 \pm 26.73$ & 777 & $43.17 \pm 36.06$ & 1484 & $-4.09(-5.36,-2.82)$ & $<0.001$ \\
\hline & Time effect & & $p<0.001^{c}$ & & $p=0.738^{c}$ & & & \\
\hline
\end{tabular}

Abbreviations: T1pp: Assessment 6-8 weeks postpartum; T2pp: Assessment one year postpartum; SD: Standard deviation; CI: Confidence interval. ${ }^{a}$ The total of participant numbers varies due to the applied covariates. ${ }^{b}$ Linear regression models fit using generalised estimating equations adjusted for pre-pregnancy BMI category, age, parity, baseline assessment and time interval between questionnaire completion date and birth date of the child. ${ }^{c}$ Linear mixed models for repeated measures adjusted for pre-pregnancy Body Mass Index (BMI) category, age and parity.

\subsubsection{Food Preparation and Dietary Choices}

Specific food preparation and dietary choices in the IG and CG are summarised in Table 3. It was not observed at T1pp, but one year after delivery, that a higher proportion of women in the IG chose whole grain bread compared to the CG. Women in the IG were more likely to use rapeseed oil and olive oil over other oils to prepare meat and fish throughout the postpartum period (T1pp: $p=0.004 ; \mathrm{T} 2 \mathrm{pp}: p=0.011$ ) and to prepare vegetables at T1pp $(p=0.012)$. The number of participants who prepared meals from fresh food at least five times a week increased in both groups from $6-8$ weeks to one year postpartum $(p<0.001$ in both groups). The proportion of vegetarians was comparable between groups. 
Table 3. Food preparation and dietary choices in the intervention and control groups.

\begin{tabular}{|c|c|c|c|c|c|c|c|c|}
\hline & \multirow{2}{*}{ Time Point } & \multicolumn{2}{|c|}{ Intervention Group } & \multicolumn{2}{|c|}{ Control Group } & \multirow{2}{*}{$n^{\mathrm{a}}$} & \multirow{2}{*}{$\begin{array}{l}\text { Adjusted OR } \\
(95 \% \mathrm{CI})\end{array}$} & \multirow{2}{*}{$\begin{array}{l}\text { Adjusted } \\
p \text { Value }^{\mathrm{b}}\end{array}$} \\
\hline & & $n$ & $\%$ & $n$ & $\%$ & & & \\
\hline \multirow{3}{*}{ Whole grain bread } & T1pp & $828 / 893$ & $92.7 \%$ & $808 / 888$ & $91.0 \%$ & 1657 & $1.21(0.65,2.28)$ & 0.546 \\
\hline & T2pp & $745 / 788$ & $94.5 \%$ & $724 / 774$ & $93.5 \%$ & 1471 & $1.46(1.07,2.01)$ & 0.017 \\
\hline & Time effect & $p=0.074^{c}$ & & $p=0.026^{c}$ & & & & \\
\hline \multirow{3}{*}{$\begin{array}{c}\text { Rapeseed oil and } \\
\text { olive oil (for meat } \\
\text { and fish) }\end{array}$} & T1pp & $456 / 729$ & $62.6 \%$ & $446 / 762$ & $58.5 \%$ & 1265 & $1.45(1.13,1.87)$ & 0.004 \\
\hline & T2pp & $397 / 615$ & $64.6 \%$ & $375 / 631$ & $59.4 \%$ & 1088 & $1.19(1.04,1.36)$ & 0.011 \\
\hline & Time effect & $p=0.535^{c}$ & & $p=0.140^{c}$ & & & & \\
\hline \multirow{3}{*}{$\begin{array}{l}\text { Rapeseed oil and } \\
\text { olive oil (for } \\
\text { vegetables) }\end{array}$} & T1pp & $496 / 748$ & $66.3 \%$ & $477 / 766$ & $62.3 \%$ & 1259 & $1.32(1.06,1.64)$ & 0.012 \\
\hline & T2pp & $447 / 653$ & $68.5 \%$ & $408 / 653$ & $62.5 \%$ & 1107 & $1.32(0.93,1.88)$ & 0.122 \\
\hline & Time effect & $p=0.215^{c}$ & & $p=0.556^{c}$ & & & & \\
\hline \multirow{3}{*}{$\begin{array}{l}\text { Cooking at least } \\
5 \text { times per week }\end{array}$} & T1pp & $536 / 893$ & $60.0 \%$ & $548 / 888$ & $61.7 \%$ & 1661 & $1.01(0.87,1.17)$ & 0.914 \\
\hline & T2pp & $578 / 771$ & $75.0 \%$ & $551 / 764$ & $72.1 \%$ & 1449 & $1.22(0.97,1.55)$ & 0.095 \\
\hline & Time effect & $p<0.001^{c}$ & & $p<0.001^{c}$ & & & & \\
\hline \multirow{3}{*}{ Vegetarian } & T1pp & $54 / 882$ & $6.1 \%$ & $47 / 886$ & $5.3 \%$ & 1677 & $1.20(0.65,2.23)$ & 0.554 \\
\hline & $\mathrm{T} 2 \mathrm{pp}$ & $44 / 789$ & $5.6 \%$ & $36 / 774$ & $4.7 \%$ & 1513 & $1.30(0.67,2.55)$ & 0.440 \\
\hline & Time effect & $p=0.717^{c}$ & & $p=0.446^{c}$ & & & & \\
\hline
\end{tabular}

Abbreviations: T1pp: Assessment 6-8 weeks postpartum; T2pp: Assessment one year postpartum; OR: Odds ratio. ${ }^{a}$ The total of participant numbers varies due to the applied covariates. ${ }^{b}$ Binary logistic regression models fit using generalised estimating equations adjusted for pre-pregnancy BMI category, age, parity, baseline assessment and time interval between questionnaire completion date and birth date of the child. ${ }^{c}$ Linear mixed models for repeated measures adjusted for pre-pregnancy BMI category, age and parity.

\subsubsection{Energy and Macronutrient Intake}

There was no significant evidence of differences between the groups in terms of mean energy and macronutrient intake (Table 4). Mean energy intake was $2236.99 \mathrm{kcal} /$ day in the IG and $2227.84 \mathrm{kcal} /$ day in the CG at T1pp. From T1pp to T2pp, the mean energy intake significantly decreased in both groups ( $p<0.001$ respectively). Alcohol consumption increased in both groups from early postpartum to one year postpartum; however, women in the IG consumed less alcohol than women in the CG one year after delivery $(p=0.014)$. The dietary quality, assessed by the HEI, was higher by trend in the IG at 6-8 weeks postpartum $(p=0.093)$. At one year postpartum, the HEI was significantly higher in the IG than in the CG $(p=0.043)$. Additional analyses revealed significant differences between the IG and CG in the following subgroups at T2pp: Women aged 26-35 years $(p=0.013)$, women with intermediate secondary education $(p<0.001)$ and multiparous women $(p=0.005)$ (Supplementary Materials Table S1). Overall, dietary quality slightly increased in both groups over time (Table 4 ).

Table 4. Mean energy and macronutrient intake in the intervention and control groups.

\begin{tabular}{|c|c|c|c|c|c|c|c|c|}
\hline & \multirow{2}{*}{ Time Point } & \multicolumn{2}{|c|}{ Intervention Group } & \multicolumn{2}{|c|}{ Control Group } & \multirow{2}{*}{$n^{\mathrm{a}}$} & \multirow{2}{*}{$\begin{array}{l}\text { Adjusted Effect } \\
\text { Size }^{b}(95 \% \text { CI })\end{array}$} & \multirow{2}{*}{$\begin{array}{l}\text { Adjusted } \\
p \text { Value }^{b}\end{array}$} \\
\hline & & $n$ & Mean \pm SD & $n$ & Mean \pm SD & & & \\
\hline \multirow{3}{*}{$\begin{array}{c}\text { Energy } \\
\text { (kcal/day) }\end{array}$} & T1pp & 838 & $2236.99 \pm 748.68$ & 824 & $2227.84 \pm 717.31$ & 1440 & $1.55(-93.15,96.26)$ & 0.974 \\
\hline & $\mathrm{T} 2 \mathrm{pp}$ & 736 & $2084.14 \pm 645.78$ & 706 & $2083.67 \pm 693.66$ & 1273 & $17.90(-28.64,64.43)$ & 0.451 \\
\hline & Time effect & & $p<0.001^{c}$ & & $p<0.001^{\mathrm{c}}$ & & & \\
\hline \multirow{3}{*}{$\begin{array}{l}\text { Carbohydrates } \\
\text { (E\%) }\end{array}$} & T1pp & 838 & $46.18 \pm 8.50$ & 824 & $46.77 \pm 9.30$ & 1440 & $-0.26(-1.60,1.07)$ & 0.701 \\
\hline & $\mathrm{T} 2 \mathrm{pp}$ & 736 & $45.62 \pm 9.32$ & 706 & $46.32 \pm 9.34$ & 1273 & $-0.80(-1.91,0.32)$ & 0.162 \\
\hline & Time effect & & $p=0.084^{c}$ & & $p=0.424^{c}$ & & & \\
\hline \multirow{3}{*}{$\begin{array}{l}\text { Saccharose } \\
\text { (g/day) }\end{array}$} & T1pp & 838 & $53.85 \pm 28.91$ & 824 & $54.34 \pm 30.53$ & 1440 & $1.83(0.89,2.76)$ & $<0.001$ \\
\hline & $\mathrm{T} 2 \mathrm{pp}$ & 736 & $45.15 \pm 24.43$ & 706 & $46.12 \pm 25.74$ & 1273 & $-0.15(-2.06,1.77)$ & 0.882 \\
\hline & Time effect & & $p<0.001^{c}$ & & $p<0.001^{\mathrm{c}}$ & & & \\
\hline
\end{tabular}


Table 4. Cont.

\begin{tabular}{|c|c|c|c|c|c|c|c|c|}
\hline & \multirow{2}{*}{ Time Point } & \multicolumn{2}{|c|}{ Intervention Group } & \multicolumn{2}{|c|}{ Control Group } & \multirow{2}{*}{$n^{\mathrm{a}}$} & \multirow{2}{*}{$\begin{array}{l}\text { Adjusted Effect } \\
\text { Size }^{b}(95 \% \text { CI })\end{array}$} & \multirow{2}{*}{$\begin{array}{l}\text { Adjusted } \\
p \text { Value }^{\mathrm{b}}\end{array}$} \\
\hline & & $n$ & Mean $\pm S D$ & $n$ & Mean \pm SD & & & \\
\hline \multirow{3}{*}{ Fibre (g/day) } & T1pp & 838 & $21.78 \pm 9.51$ & 824 & $20.98 \pm 9.09$ & 1440 & $0.34(-0.62,1.29)$ & 0.491 \\
\hline & $\mathrm{T} 2 \mathrm{pp}$ & 736 & $21.63 \pm 8.95$ & 706 & $20.86 \pm 8.93$ & 1273 & $0.55(-0.24,1.35)$ & 0.172 \\
\hline & Time effect & & $p<0.001^{\mathrm{c}}$ & & $p<0.001^{\mathrm{c}}$ & & & \\
\hline \multirow{3}{*}{ Fat $(\mathrm{E} \%)$} & T1pp & 838 & $35.04 \pm 7.49$ & 824 & $34.55 \pm 7.80$ & 1440 & $0.23(-0.93,1.39)$ & 0.698 \\
\hline & $\mathrm{T} 2 \mathrm{pp}$ & 736 & $34.77 \pm 8.09$ & 706 & $34.35 \pm 7.66$ & 1273 & $0.38(-0.52,1.27)$ & 0.411 \\
\hline & Time effect & & $p=0.230^{c}$ & & $p=0.096^{c}$ & & & \\
\hline \multirow{3}{*}{ Protein $(\mathrm{E} \%)$} & T1pp & 838 & $18.57 \pm 4.07$ & 824 & $18.40 \pm 4.51$ & 1440 & $0.17(-0.13,0.47)$ & 0.262 \\
\hline & T2pp & 736 & $18.86 \pm 4.25$ & 706 & $18.48 \pm 4.23$ & 1273 & $0.46(0.05,0.86)$ & 0.028 \\
\hline & Time effect & & $p=0.017^{c}$ & & $p=0.253^{c}$ & & & \\
\hline \multirow{3}{*}{$\begin{array}{l}\text { Alcohol } \\
\text { (g/day) }\end{array}$} & T1pp & 838 & $0.55 \pm 1.57$ & 824 & $0.79 \pm 2.72$ & 1440 & $-0.24(-0.58,0.10)$ & 0.159 \\
\hline & $\mathrm{T} 2 \mathrm{pp}$ & 736 & $2.03 \pm 2.59$ & 706 & $2.34 \pm 3.62$ & 1273 & $-0.27(-0.49,-0.06)$ & 0.014 \\
\hline & Time effect & & $p<0.001^{c}$ & & $p<0.001^{c}$ & & & \\
\hline \multirow{3}{*}{ HEI } & T1pp & 898 & $55.82 \pm 8.37$ & 893 & $54.81 \pm 8.38$ & 1676 & $0.64(-0.11,1.39)$ & 0.093 \\
\hline & $\mathrm{T} 2 \mathrm{pp}$ & 791 & $56.22 \pm 8.78$ & 777 & $55.11 \pm 8.33$ & 1485 & $0.85(0.03,1.68)$ & 0.043 \\
\hline & Time effect & & $p<0.001^{c}$ & & $p<0.001^{\mathrm{c}}$ & & & \\
\hline
\end{tabular}

Abbreviations: HEI: Healthy Eating Index; T1pp: Assessment 6-8 weeks postpartum; T2pp: Assessment one year postpartum; SD: Standard deviation; CI: confidence interval. ${ }^{a}$ The total of participant numbers varies due to the applied covariates. ${ }^{b}$ Linear regression models fit using generalised estimating equations adjusted for pre-pregnancy BMI category, age, parity, baseline intake and time interval between questionnaire completion date and birth date of the child. ${ }^{c}$ Linear mixed models for repeated measures adjusted for pre-pregnancy BMI category, age and parity.

\subsection{Postpartum Physical Activity Behaviour}

Maternal postpartum PA behaviour is presented in Table 5. The level of total PA was slightly higher in the CG at T1pp compared to the IG $(p=0.023)$. Subgroup analyses (Tables S2-S4) revealed a lower total PA in the IG compared to the CG in the subgroup of women aged 18-25 years $(p=0.044)$, the subgroup of women with normal weight $(p=0.047)$ and the subgroup of primiparous women $(p=0.001)$ (Table S2). Over the course of the postpartum period, there was a significant increase in total PA in the IG $(p<0.001)$, but not in the CG $(p=0.061)$, resulting in a similar mean PA level in both groups one year after delivery (Table 5). Women in the IG showed a significantly higher level of occupational activity compared to the CG at T1pp $(p=0.016)$. No between-group differences in intensity of PA or other activity categories, including sport activity, were observed. In both groups, the mean MET-h/week in sedentary activities and the level of inactivity decreased significantly during the postpartum period ( $p<0.001$ respectively). In most other PA categories, levels increased significantly within the first year after delivery in both groups. The between-group differences in meeting the PA recommendations at T1pp (IG: 52.7\%, CG: 46.7\%, $p=0.060$ ) and at T2pp (IG: 58.1\%, CG: 55.2\%, $p=0.957$ ) were not statistically significant.

Table 5. Postpartum physical activity behaviour in the intervention and control groups.

\begin{tabular}{|c|c|c|c|c|c|c|c|c|}
\hline & \multirow{2}{*}{ Time Point } & \multicolumn{2}{|c|}{ Intervention Group } & \multicolumn{2}{|c|}{ Control Group } & \multirow{2}{*}{$n^{\mathrm{a}}$} & \multirow{2}{*}{$\begin{array}{l}\text { Adjusted Effect } \\
\text { Size }^{b}(95 \% \text { CI })\end{array}$} & \multirow{2}{*}{$\begin{array}{l}\text { Adjusted } \\
p \text { Value }\end{array}$} \\
\hline & & $n$ & Mean \pm SD & $n$ & Mean \pm SD & & & \\
\hline \multicolumn{9}{|c|}{ Total PA (MET-h/week) } \\
\hline \multirow{3}{*}{ Total PA } & T1pp & 853 & $179.0 \pm 63.9$ & 863 & $183.0 \pm 68.8$ & 1538 & $-3.59(-6.69,-0.50)$ & 0.023 \\
\hline & T2pp & 724 & $189.5 \pm 64.7$ & 737 & $188.1 \pm 70.9$ & 1338 & $2.60(-5.23,10.43)$ & 0.515 \\
\hline & Time effect & & $p<0.001^{c}$ & & $p=0.061^{c}$ & & & \\
\hline \multirow{3}{*}{$\begin{array}{c}\text { Total PA of light } \\
\text { intensity and } \\
\text { above }\end{array}$} & T1pp & 856 & $168.9 \pm 62.2$ & 866 & $172.3 \pm 67.5$ & 1552 & $-2.58(-5.56,0.40)$ & 0.090 \\
\hline & $\mathrm{T} 2 \mathrm{pp}$ & 725 & $182.2 \pm 63.8$ & 740 & $180.6 \pm 70.1$ & 1348 & $2.71(-4.95,10.37)$ & 0.488 \\
\hline & Time effect & & $p<0.001^{\mathrm{c}}$ & & $p<0.001^{\mathrm{c}}$ & & & \\
\hline
\end{tabular}


Table 5. Cont.

\begin{tabular}{|c|c|c|c|c|c|c|c|c|}
\hline & \multirow{2}{*}{ Time Point } & \multicolumn{2}{|c|}{ Intervention Group } & \multicolumn{2}{|c|}{ Control Group } & \multirow{2}{*}{$n^{\mathrm{a}}$} & \multirow{2}{*}{$\begin{array}{l}\text { Adjusted Effect } \\
\text { Size }^{b}(95 \% \text { CI })\end{array}$} & \multirow{2}{*}{$\begin{array}{l}\text { Adjusted } \\
p \text { Value }^{\text {b }}\end{array}$} \\
\hline & & $n$ & Mean \pm SD & $n$ & Mean \pm SD & & & \\
\hline \multicolumn{9}{|c|}{ Intensity (MET-h/week) } \\
\hline \multirow{3}{*}{ Sedentary } & T1pp & 903 & $10.1 \pm 10.0$ & 899 & $10.7 \pm 10.3$ & 1664 & $-0.90(-1.83,0.03)$ & 0.058 \\
\hline & $\mathrm{T} 2 \mathrm{pp}$ & 773 & $7.1 \pm 5.9$ & 759 & $7.6 \pm 6.6$ & 1446 & $-0.49(-1.02,0.05)$ & 0.077 \\
\hline & Time effect & & $p<0.001^{\mathrm{c}}$ & & $p<0.001^{\mathrm{c}}$ & & & \\
\hline \multirow{3}{*}{ Light-intensity } & T1pp & 890 & $87.6 \pm 33.4$ & 885 & $90.8 \pm 33.7$ & 1628 & $-1.37(-5.08,2.34)$ & 0.469 \\
\hline & $\mathrm{T} 2 \mathrm{pp}$ & 753 & $105.4 \pm 34.4$ & 755 & $103.5 \pm 34.4$ & 1407 & $1.90(-1.17,4.97)$ & 0.224 \\
\hline & Time effect & & $p<0.001^{\mathrm{c}}$ & & $p<0.001^{c}$ & & & \\
\hline \multirow{3}{*}{$\begin{array}{l}\text { Moderate- } \\
\text { intensity }\end{array}$} & T1pp & 867 & $80.5 \pm 41.1$ & 881 & $80.4 \pm 43.5$ & 1588 & $0.84(-0.23,1.92)$ & 0.124 \\
\hline & $\mathrm{T} 2 \mathrm{pp}$ & 746 & $74.9 \pm 43.0$ & 746 & $75.1 \pm 47.9$ & 1387 & $1.00(-3.35,5.36)$ & 0.651 \\
\hline & Time effect & & $p<0.001^{\mathrm{c}}$ & & $p=0.001^{c}$ & & & \\
\hline \multirow{3}{*}{$\begin{array}{l}\text { Vigorous- } \\
\text { intensity }\end{array}$} & T1pp & 901 & $1.2 \pm 3.3$ & 902 & $1.0 \pm 3.3$ & 1671 & $0.20(-0.09,0.50)$ & 0.172 \\
\hline & $\mathrm{T} 2 \mathrm{pp}$ & 774 & $2.3 \pm 4.7$ & 766 & $2.0 \pm 4.6$ & 1456 & $0.32(-0.14,0.78)$ & 0.170 \\
\hline & Time effect & & $p<0.001^{\mathrm{c}}$ & & $p<0.001^{\mathrm{c}}$ & & & \\
\hline \multicolumn{9}{|c|}{ Type (MET-h/week) } \\
\hline \multirow{3}{*}{$\begin{array}{l}\text { Household } \\
\text { activity }\end{array}$} & T1pp & 882 & $141.2 \pm 55.2$ & 887 & $145.1 \pm 60.0$ & 1625 & $-1.75(-7.54,4.04)$ & 0.553 \\
\hline & $\mathrm{T} 2 \mathrm{pp}$ & 749 & $140.8 \pm 54.2$ & 751 & $137.6 \pm 55.3$ & 1402 & $4.13(-2.23,10.50)$ & 0.203 \\
\hline & Time effect & & $p=0.880^{c}$ & & $p<0.001^{\mathrm{c}}$ & & & \\
\hline \multirow{3}{*}{$\begin{array}{l}\text { Occupational } \\
\text { activity }\end{array}$} & T1pp & 48 & $24.1 \pm 38.9$ & 52 & $18.6 \pm 24.5$ & 83 & $8.09(1.51,14.67)$ & 0.016 \\
\hline & T2pp & 218 & $42.1 \pm 33.1$ & 218 & $44.8 \pm 36.2$ & 365 & $-2.17(-8.23,3.88)$ & 0.482 \\
\hline & Time effect & & $p=0.002^{\mathrm{c}}$ & & $p<0.001^{c}$ & & & \\
\hline \multirow{3}{*}{ Sport activity } & T1pp & 884 & $9.7 \pm 8.2$ & 888 & $9.2 \pm 9.2$ & 1627 & $0.12(-0.55,0.79)$ & 0.725 \\
\hline & $\mathrm{T} 2 \mathrm{pp}$ & 756 & $12.5 \pm 10.9$ & 753 & $11.4 \pm 10.5$ & 1417 & $0.60(-1.28,2.47)$ & 0.532 \\
\hline & Time effect & & $p<0.001^{c}$ & & $p<0.001^{c}$ & & & \\
\hline \multirow{3}{*}{$\begin{array}{c}\text { Transportation } \\
\text { activity }\end{array}$} & T1pp & 902 & $14.3 \pm 13.4$ & 899 & $14.2 \pm 13.4$ & 1661 & $0.41(-0.79,1.61)$ & 0.505 \\
\hline & $\mathrm{T} 2 \mathrm{pp}$ & 768 & $14.9 \pm 12.9$ & 762 & $15.2 \pm 13.6$ & 1439 & $0.01(-1.09,1.11)$ & 0.986 \\
\hline & Time effect & & $p=0.323^{c}$ & & $p=0.099^{c}$ & & & \\
\hline \multirow{3}{*}{ Inactivity } & T1pp & 900 & $12.7 \pm 11.5$ & 896 & $13.2 \pm 11.3$ & 1657 & $-0.75(-1.85,0.35)$ & 0.182 \\
\hline & $\mathrm{T} 2 \mathrm{pp}$ & 771 & $9.6 \pm 7.6$ & 757 & $10.3 \pm 8.3$ & 1441 & $-0.63(-1.35,0.10)$ & 0.091 \\
\hline & Time effect & & $p<0.001^{\mathrm{c}}$ & & $p<0.001^{c}$ & & & \\
\hline \multicolumn{6}{|c|}{ Meeting PA recommendations $(n(\%))^{\mathrm{d}}$} & & OR $(95 \% \mathrm{CI})^{\mathrm{e}}$ & \\
\hline & T1pp & $466 / 884$ & $52.7 \%$ & $415 / 888$ & $46.7 \%$ & 1691 & $1.15(0.99,1.33)$ & 0.060 \\
\hline & $\mathrm{T} 2 \mathrm{pp}$ & $439 / 756$ & $58.1 \%$ & $416 / 753$ & $55.2 \%$ & 1467 & $1.01(0.83,1.21)$ & 0.957 \\
\hline
\end{tabular}

Abbreviations: T1pp: Assessment 6-8 weeks postpartum; T2pp: Assessment one year postpartum; SD: Standard deviation; CI: Confidence interval; PA: Physical activity; OR: Odds ratio. ${ }^{a}$ The total of participant numbers varies due to the applied covariates. ${ }^{b}$ Linear regression models fit using generalised estimating equations adjusted for pre-pregnancy BMI category, age, parity, baseline assessment and time interval between questionnaire completion date and birth date of the child. ${ }^{\mathrm{c}}$ Linear mixed models for repeated measures adjusted for pre-pregnancy BMI category, age and parity. ${ }^{\mathrm{d}}$ Meeting recommendations defined as $\geq 7.5 \mathrm{MET}-\mathrm{h} /$ week in category sports activity of moderate-intensity or greater. ${ }^{\mathrm{e}}$ Binary logistic regression models fit using generalised estimating equations adjusted for pre-pregnancy BMI category, age, parity, baseline assessment and time interval between questionnaire completion date and birth date of the child.

\subsection{Maternal Smoking Behaviour}

Figure 2 depicts the smoking behaviour in the IG and the CG from early pregnancy until one year postpartum. At baseline, smoking rate was $5.0 \%$ in each group. While the proportion of smokers in the CG did not change over the course of pregnancy, it decreased in the IG, so that significantly fewer women in the IG smoked in late pregnancy (IG: $3.8 \%$ vs. CG: $5.1 \%, p<0.001)$. After delivery, the proportion of smokers increased in both groups. Nonetheless, at $6-8$ weeks as well as at one year postpartum, the smoking rate was significantly lower in the IG compared to the CG (T1pp: $7.1 \%$ vs. $9.7 \%, p<0.001$; T2pp: $13.1 \%$ vs. $14.1 \%, p<0.001)$. At both postpartum time points, the number of current smokers in the IG was consistently lower in the subgroup of women with normal BMI 
(T1pp: $p=0.019$; T2pp: $p=0.017)$ and of women who went to intermediate secondary school (T1pp: $p<0.001$; T2pp: $p<0.001$ ) (Table S5). Post-hoc interaction analysis revealed that educational level influenced the relationship between the intervention and smoking behaviour one year after delivery $(p=0.042)$.

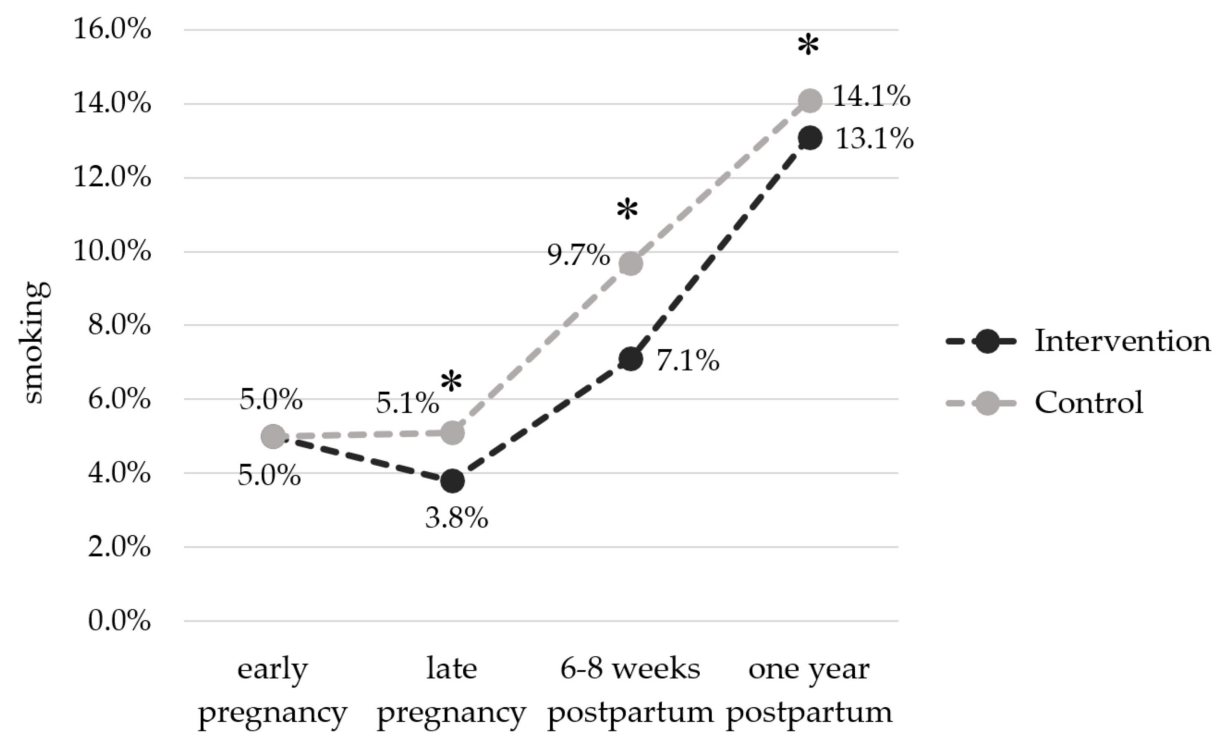

Figure 2. Percentage of smokers in the intervention and control groups from pregnancy to one year postpartum. Early pregnancy: IG: $n=47 / 931$, CG: $n=46 / 922$; late pregnancy: IG: $n=35 / 916$, CG: $n=46 / 906$; 6-8 weeks postpartum: IG: $n=64 / 905, C G: n=88 / 910$; one year postpartum: IG: $n=103 / 785$, CG: $n=109 / 772$. ${ }^{*} p<0.001$.

\section{Discussion}

The aim of this secondary analysis was to examine whether the GeliS lifestyle intervention had a lasting effect on maternal health behaviour during the first year postpartum. The findings of the present analysis indicate that the GeliS intervention successfully improved some aspects of maternal postpartum health behaviour.

Women who had received lifestyle counselling showed a lower mean intake of fast food and soft drinks and consumed more vegetables than women in the CG. Furthermore, women in the IG were more likely to choose healthy oils for food preparation, and the overall quality of their diet assessed by the HEI was higher. In contrast, no difference was detected for total energy intake. This is in line with the observed intervention effect on women's antenatal dietary behaviour [42] and suggests a sustained effect in the postpartum period. Since some differences in dietary behaviour were small, it is questionable if their clinical relevance is meaningful. Irrespective of group allocation, the overall dietary quality and vegetable intake increased and the consumption of sweets and snacks decreased during the postpartum phase. This corresponds to observations from Martin et al. [29], who also reported that dietary quality improved in the late vs. the early postpartum phase. Possible reasons for this might be the unique challenges present during the early postpartum period, such as fragmented sleep [54], fatigue [28] and prioritising the needs of the infant [27]. Due to childcare responsibilities and limited time after birth, healthy eating might not be a priority [28] and women might return to previous habits, disregarding healthy lifestyle advice [27].

Although some improvements in PA were evident during pregnancy [41], only minor between-group differences were found for the postpartum period. For instance, while a significantly higher proportion of women in the IG met the PA recommendations in late pregnancy [41], this significance disappeared in the postpartum period. A similar change was observed by Sanda et al. [55]. Nevertheless, only women in the IG increased total PA significantly over the course of the first postpartum year. Despite several minor 
trends towards a beneficial intervention effect, our data suggests that the GeliS lifestyle intervention was not successful in comprehensively influencing the PA behaviour beyond delivery. Irrespective of group allocation, the observed increase in TALIA over the postpartum period corresponds with previous observational studies in pregnant women $[31,56]$. Further, the proportion of women in the IG and the CG who met the PA recommendations in the postpartum period (T1pp: $52.7 \%$ vs. $46.7 \%$; T2pp: $58.1 \%$ vs. $55.2 \%$ ) exceeded the proportion of women meeting PA guidelines in the general German population (42.6\%) measured by means of a written form of the European Health Interview Survey-Physical Activity Questionnaire [57]. The discrepancy with our data might be partly explained by the difficulty in estimating PA levels with the PPAQ. In both groups, the largest increase in PA levels from pregnancy to postpartum was observed for household activities, equally reported by Dodd et al. [25], and might be attributed to increasing caregiving tasks, which are not separately assessed by the PPAQ.

We further observed a lower proportion of women who smoked in the IG compared to the CG during late pregnancy and the postpartum period. This is in contrast to the findings from a systematic review and meta-analysis [58]. Chamberlain et al. concluded that there is uncertainty if lifestyle interventions focusing not only on smoking, but also on enhancing maternal health overall, increase the chances of smoking cessation [58]. The smoking rate in our cohort was lower compared to data from a German cohort on smoking rates during pregnancy (10.9\%) [59], and the general female adult population $(20.8 \%)$ [60]. This discrepancy might be explained by a reporting bias or the data collection mode via questionnaires. Nevertheless, our subgroup analysis corresponds with these German-wide data indicating that smoking is less prevalent among adults with a higher educational level and most common in younger age groups [60]. In conclusion, the GeliS lifestyle intervention led to moderate beneficial effects on maternal health behaviours during pregnancy $[41,42]$, which were partly sustained in the postpartum period.

Results from previous lifestyle intervention studies are inconclusive, and few randomised controlled trials (RCT) have investigated the sustained effects of mixed lifestyle interventions on maternal lifestyle behaviours up to 12 months postpartum. In contrast to our data, which include women with different BMI categories, two comparable large-scale RCTs recruited only women with overweight and obesity [25,33,34]. In the LIMIT study, many of the improvements in dietary quality and PA achieved during pregnancy were not sustained at four months postpartum [25]. In the UPBEAT trial, the positive changes in dietary behaviour observed in pregnant women with obesity persisted at six months [34] and three years postpartum [33]. However, no lasting effect on PA was achieved [33,34]. These results are, to some extent, in line with our observations. The Danish LiP-study found no effects on self-reported PA and eating habits among women with obesity at six months postpartum after receiving exercise classes during pregnancy [61]. In contrast, the ROLO trial reported continued compliance on dietary behaviours at three months postpartum [35]. In the aforementioned studies, follow-up assessment time points ranged from three months to three years [25,33-35,61], complicating the ability to determine whether intervention effects ultimately persist in the long-term. Altogether, findings from relevant studies depict a heterogeneous picture with respect to study design, population characteristics and type of intervention, which make direct comparisons to our data challenging. In contrast to the above-mentioned studies, our study was the only one that was implemented in a primary care setting.

Several limitations of our analysis should be considered. Delivering the lifestyle counselling within the primary care system implied that the intervention was carried out by trained medical staff rather than lifestyle experts, e.g., dieticians or physiotherapists. Furthermore, our analyses largely relied on self-reported data from questionnaires. Variations in the timing of completion of the questionnaires is a consideration, and was controlled for by including the completion date as a confounding variable. Moreover, self-reported data are susceptible to inaccuracies, despite our efforts to reduce over- or underreporting. However, the applied DEGS-FFQ is a validated tool and especially useful for comparing 
dietary intake between groups [47]. While the PPAQ is a validated tool for the assessment of PA during pregnancy [49], it has not been formally validated for the postpartum period. Nevertheless, using the same questionnaires pre- and postnatally enabled us to compare results at different time points.

Apart from these limitations, our study has several strengths that are worth mentioning. Importantly, the GeliS study was conceived and implemented as a public health RCT imbedded in routine care. The unique study design allowed the recruitment of a large cohort, comprising initially 2286 women, as the intervention was able to be delivered during pre- and post-natal visits. For this follow-up analysis, we included data from 1899 women, representing $83.7 \%$ of the original cohort. Compared to other studies $[34,62]$, this is an exceptionally high retention rate. To the best of our knowledge, GeliS is the first large-scale trial that was conducted in a routine care setting that showed a detailed analysis of health behaviours of women in different BMI categories during the first year postpartum.

This analysis emphasises the need for future research on maternal health after delivery. In view of the challenge of postpartum weight retention [6,9], understanding key determinants of maintaining and even increasing healthy lifestyle habits during the postpartum period is a crucial public health issue. Because women face multiple barriers to adopting a healthy postpartum lifestyle [32], interventions should consider practical concerns. A technology-based approach may be able to alleviate barriers and provide tailored support within a woman's daily life [63].

\section{Conclusions}

The GeliS trial is the only study conducted in a routine care setting presenting comprehensive information on maternal health behaviour up to 12 months postpartum. While previous results of the GeliS trial indicated that the lifestyle intervention was able to improve dietary and PA behaviour during pregnancy [41,42], this secondary analysis still showed slightly positive intervention effects on maternal health behaviour beyond the intervention phase. More attention should be given to a healthy postnatal lifestyle to mitigate the long-term obesity risk and related co-morbidities. More high-quality studies are needed to clarify the remaining uncertainty regarding the optimal approach to support a healthy postpartum lifestyle. Data on GeliS mother-child pairs up to the children's fifth birthday will offer the opportunity to investigate whether differences in maternal postpartum lifestyle also have an impact on the children's health behaviours and health-related outcomes.

Supplementary Materials: The following are available online at https://www.mdpi.com/article/10 .3390/nu13041310/s1, Table S1: Healthy Eating Index in subgroups of the intervention and control groups. Table S2: Total physical activity in subgroups of the intervention and control groups. Table S3: Meeting physical activity recommendations in subgroups of the intervention and control groups. Table S4: Sedentary activity in subgroups of the intervention and control groups. Table S5: Smoking in subgroups of the intervention and control groups.

Author Contributions: Conceptualisation and methodology, H.H., K.R., J.H., J.G., M.S. and K.G.; formal analysis, J.H., J.G., M.S. and K.G.; investigation, K.R., J.H. and J.G.; resources, K.R. and J.G.; data curation, M.S., K.G.; writing-original draft preparation, M.S. and K.G.; writing-review and editing, J.H., J.G., R.R., D.M. and H.H.; visualization, M.S. and K.G.; supervision, H.H.; project administration, H.H. and K.R.; funding acquisition, H.H. All authors have read and agreed to the published version of the manuscript.

Funding: The study was funded by the Else Kröner-Fresenius Foundation, Bad Homburg, Germany (Grant Number: 5140889), the Competence Centre for Nutrition (KErn) in Bavaria, the Bavarian State Ministry of Food, Agriculture and Forestry, the Bavarian State Ministry of Health and Care (Health Initiative "Gesund.Leben.Bayern."), the AOK Bayern, the largest statutory health insurance in Bavaria, as well as the DEDIPAC consortium by the Joint Programming Initiative (JPI) "A Healthy Diet for a Healthy Life". 
Institutional Review Board Statement: The study was conducted according to the guidelines of the Declaration of Helsinki and the study protocol was approved by the Ethics Committee of the Technical University of Munich (project number 5653/13, 5 February 2013).

Informed Consent Statement: Informed consent was obtained from all subjects involved in the study.

Data Availability Statement: The datasets used and analysed during the current study are available from the corresponding author on reasonable request.

Acknowledgments: We gratefully acknowledge the valuable contribution from our partners and funders, the Competence Centre for Nutrition, the Bavarian State Ministry of Food, Agriculture and Forestry, the Bavarian State Ministry of Health and Care, the AOK Bayern and the Else KrönerFresenius foundation, Bad Homburg, Germany. Moreover, we gratefully thank all cooperation partners and the expert advisory board who have been named and acknowledged elsewhere [39]. In particular, we would like to thank Gert Mensink, Robert Koch Institute, Berlin, for guidance in the analysis of the FFQ including the Healthy Eating Index, and the Max Rubner Institute, Karlsruhe, for provision of reference data from the German National Consumption Survey II (NVS II). Furthermore, we would like to thank Lynne Stecher for statistical support within the GeliS study. Finally, we would like to thank our colleagues and former colleagues from the Institute for Nutritional Medicine at the Technical University of Munich Julia Kunath, Christina Holzapfel, Isabel Lück and Annie Naujoks, and the Bavarian Competence Centre for Nutrition, Eva Rosenfeld and Luzia Kick for their support, and all participating practices, gynaecologists, medical personnel, midwives, participants and their families for their involvement.

Conflicts of Interest: The authors declare no conflict of interest. The funders had no role in the design of the study; in the collection, analyses, or interpretation of data; in the writing of the manuscript, or in the decision to publish the results.

$\begin{array}{ll}\text { Abbreviations } \\ \text { BMI } & \text { Body mass index } \\ \text { CG } & \text { Control group } \\ \text { CI } & \text { Confidence interval } \\ \text { DEGS } & \text { German Health Interview and Examination Survey for Adults } \\ \text { DEGS-HEI } & \text { DEGS-Healthy Eating Index } \\ \text { FFQ } & \text { Food frequency questionnaire } \\ \text { GEE } & \text { Generalised estimating equations } \\ \text { GeliS } & \text { "Gesund leben in der Schwangerschaft" / Healthy living in pregnancy" } \\ \text { GWG } & \text { Gestational weight gain } \\ \text { HEI } & \text { Healthy Eating Index } \\ \text { IG } & \text { Intervention group } \\ \text { IOM } & \text { Institute of Medicine } \\ \text { MET } & \text { Metabolic equivalent of task } \\ \text { OR } & \text { Odds ratio } \\ \text { PA } & \text { Physical activity } \\ \text { RCT } & \text { Randomised controlled trial } \\ \text { SD } & \text { Standard deviation } \\ \text { T1pp } & \text { 6-8 weeks postpartum (time point of first data collection postpartum) } \\ \text { T2pp } & \text { One year postpartum (time point of second data collection postpartum) } \\ \text { PPAQ } & \text { Pregnancy Physical Activity Questionnaire }\end{array}$

\section{References}

1. Pereira, M.A.; Rifas-Shiman, S.L.; Kleinman, K.P.; Rich-Edwards, J.W.; Peterson, K.E.; Gillman, M.W. Predictors of change in physical activity during and after pregnancy: Project Viva. Am. J. Prev. Med. 2007, 32, 312-319. [CrossRef]

2. Kopp, W. How Western Diet And Lifestyle Drive The Pandemic Of Obesity And Civilization Diseases. Diabetes Metab. Syndr. Obes. 2019, 12, 2221-2236. [CrossRef]

3. Seaman, D.R. Weight gain as a consequence of living a modern lifestyle: A discussion of barriers to effective weight control and how to overcome them. J. Chiropr. Humanit. 2013, 20, 27-35. [CrossRef] 
4. Noever, K.; Schubert, J.; Reuschel, E.; Timmesfeld, N.; Arabin, B. Changes in Maternal Body Mass Index, Weight Gain and Outcome of Singleton Pregnancies from 2000 to 2015: A Population-based Retrospective Cohort Study in Hesse/Germany. Geburtshilfe Frauenheilkd. 2020, 80, 508-517. [CrossRef]

5. Institute of Medicine (US) and National Research Council (US) Committee to Reexamine IOM Pregnancy Weight Guidelines. Weight Gain during Pregnancy: Reexamining the Guidelines; Rasmussen, K.M., Yaktine, A.L., Eds.; National Academies Press: Washington, DC, USA, 2009; ISBN 9780309131131.

6. $\quad$ Endres, L.K.; Straub, H.; McKinney, C.; Plunkett, B.; Minkovitz, C.S.; Schetter, C.D.; Ramey, S.; Wang, C.; Hobel, C.; Raju, T.; et al. Postpartum weight retention risk factors and relationship to obesity at 1 year. Obstet. Gynecol. 2015, 125, 144-152. [CrossRef]

7. Nehring, I.; Schmoll, S.; Beyerlein, A.; Hauner, H.; von Kries, R. Gestational weight gain and long-term postpartum weight retention: A meta-analysis. Am. J. Clin. Nutr. 2011, 94, 1225-1231. [CrossRef]

8. Hoffmann, J.; Günther, J.; Stecher, L.; Spies, M.; Meyer, D.; Kunath, J.; Raab, R.; Rauh, K.; Hauner, H. Effects of a Lifestyle Intervention in Routine Care on Short- and Long-Term Maternal Weight Retention and Breastfeeding Behavior-12 Months Follow-up of the Cluster-Randomized GeliS Trial. J. Clin. Med. 2019, 8, 876. [CrossRef]

9. Luke, S.; Kirby, R.S.; Wright, L. Postpartum Weight Retention and Subsequent Pregnancy Outcomes. J. Perinat. Neonatal Nurs. 2016, 34, 292-301. [CrossRef]

10. Gunderson, E.P.; Abrams, B.; Selvin, S. The relative importance of gestational gain and maternal characteristics associated with the risk of becoming overweight after pregnancy. Int. J. Obes. Relat. Metab. Disord. 2000, 24, 1660-1668. [CrossRef]

11. Livingstone, K.M.; McNaughton, S.A. Diet quality is associated with obesity and hypertension in Australian adults: A cross sectional study. BMC Public Health 2016, 16, 1037. [CrossRef]

12. Boggs, D.A.; Rosenberg, L.; Rodríguez-Bernal, C.L.; Palmer, J.R. Long-term diet quality is associated with lower obesity risk in young African American women with normal BMI at baseline. J. Nutr. 2013, 143, 1636-1641. [CrossRef] [PubMed]

13. Mottola, M.F. Pregnancy, Physical Activity and Weight Control to Prevent Obesity and Future Chronic Disease Risk in Both Mother and Child. CWHR 2015, 11, 31-40. [CrossRef]

14. Shah, B.S.; Freeland-Graves, J.H.; Cahill, J.M.; Lu, H.; Graves, G.R. Diet quality as measured by the healthy eating index and the association with lipid profile in low-income women in early postpartum. J. Am. Diet. Assoc. 2010, 110, 274-279. [CrossRef]

15. Melzer, K.; Schutz, Y.; Boulvain, M.; Kayser, B. Physical activity and pregnancy: Cardiovascular adaptations, recommendations and pregnancy outcomes. Sports Med. 2010, 40, 493-507. [CrossRef]

16. Aune, D.; Saugstad, O.D.; Henriksen, T.; Tonstad, S. Physical activity and the risk of preeclampsia: A systematic review and meta-analysis. Epidemiology 2014, 25, 331-343. [CrossRef]

17. Larson-Meyer, D.E. Effect of postpartum exercise on mothers and their offspring: A review of the literature. Obes. Res. 2002, 10, 841-853. [CrossRef]

18. Da Costa, D.; Rippen, N.; Dritsa, M.; Ring, A. Self-reported leisure-time physical activity during pregnancy and relationship to psychological well-being. J. Psychosom. Obstet. Gynaecol. 2003, 24, 111-119. [CrossRef]

19. Dipietro, L.; Evenson, K.R.; Bloodgood, B.; Sprow, K.; Troiano, R.P.; Piercy, K.L.; Vaux-Bjerke, A.; Powell, K.E. Benefits of Physical Activity during Pregnancy and Postpartum: An Umbrella Review. Med. Sci. Sports Exerc. 2019, 51, 1292-1302. [CrossRef]

20. Koletzko, B.; Godfrey, K.M.; Poston, L.; Szajewska, H.; van Goudoever, J.B.; de Waard, M.; Brands, B.; Grivell, R.M.; Deussen, A.R.; Dodd, J.M.; et al. Nutrition During Pregnancy, Lactation and Early Childhood and its Implications for Maternal and Long-Term Child Health: The Early Nutrition Project Recommendations. Ann. Nutr. Metab. 2019, 74, 93-106. [CrossRef]

21. Koletzko, B.; Bauer, C.-P.; Cierpka, M.; Cremer, M.; Flothkötter, M.; Graf, C.; Heindl, I.; Hellmers, C.; Kersting, M.; Krawinkel, M.; et al. Ernährung und Bewegung von Säuglingen und stillenden Frauen. Monatsschr. Kinderheilkd. 2016, 164, 771-798. [CrossRef]

22. Ferrari, N.; Graf, C. Bewegungsempfehlungen für Frauen während und nach der Schwangerschaft. Gesundheitswesen 2017, 79, S36-S39. [CrossRef] [PubMed]

23. The American College of Obstetricians and Gynecologists. ACOG Committee Opinion No. 650 (Reaffirmed 2019): Physical Activity and Exercise During Pregnancy and the Postpartum Period. Obstet. Gynecol. 2015, 126, e135-e142. [CrossRef]

24. Poston, L.; Bell, R.; Croker, H.; Flynn, A.C.; Godfrey, K.M.; Goff, L.; Hayes, L.; Khazaezadeh, N.; Nelson, S.M.; Oteng-Ntim, E.; et al. Effect of a behavioural intervention in obese pregnant women (the UPBEAT study): A multicentre, randomised controlled trial. Lancet Diabetes Endocrinol. 2015, 3, 767-777. [CrossRef]

25. Dodd, J.M.; Cramp, C.; Sui, Z.; Yelland, L.N.; Deussen, A.R.; Grivell, R.M.; Moran, L.J.; Crowther, C.A.; Turnbull, D.; McPhee, A.J.; et al. The effects of antenatal dietary and lifestyle advice for women who are overweight or obese on maternal diet and physical activity: The LIMIT randomised trial. BMC Med. 2014, 12, 161. [CrossRef]

26. The International Weight Management in Pregnancy (i-WIP) Collaborative Group. Effect of diet and physical activity based interventions in pregnancy on gestational weight gain and pregnancy outcomes: Meta-analysis of individual participant data from randomised trials. BMJ 2017, 358, j3119. [CrossRef]

27. Faria-Schützer, D.; Surita, F.; Rodrigues, L.; Turato, E. Eating Behaviors in Postpartum: A Qualitative Study of Women with Obesity. Nutrients 2018, 10, 885. [CrossRef] [PubMed]

28. Murray-Davis, B.; Grenier, L.; Atkinson, S.A.; Mottola, M.F.; Wahoush, O.; Thabane, L.; Xie, F.; Vickers-Manzin, J.; Moore, C.; Hutton, E.K. Experiences regarding nutrition and exercise among women during early postpartum: A qualitative grounded theory study. BMC Pregnancy Childbirth 2019, 19, 368. [CrossRef] [PubMed] 
29. Martin, J.C.; Joham, A.E.; Mishra, G.D.; Hodge, A.M.; Moran, L.J.; Harrison, C.L. Postpartum Diet Quality: A Cross-Sectional Analysis from the Australian Longitudinal Study on Women's Health. J. Clin. Med. 2020, 9, 446. [CrossRef]

30. Moran, L.J.; Sui, Z.; Cramp, C.S.; Dodd, J.M. A decrease in diet quality occurs during pregnancy in overweight and obese women which is maintained post-partum. Int. J. Obes. 2013, 37, 704-711. [CrossRef]

31. Borodulin, K.; Evenson, K.R.; Herring, A.H. Physical activity patterns during pregnancy through postpartum. BMC Women Health 2009, 9, 32. [CrossRef]

32. Carter-Edwards, L.; Østbye, T.; Bastian, L.A.; Yarnall, K.S.H.; Krause, K.M.; Simmons, T.-J. Barriers to adopting a healthy lifestyle: Insight from postpartum women. BMC Res. Notes 2009, 2, 161. [CrossRef]

33. Dalrymple, K.V.; Tydeman, F.A.S.; Taylor, P.D.; Flynn, A.C.; O’Keeffe, M.; Briley, A.L.; Santosh, P.; Hayes, L.; Robson, S.C.; Nelson, S.M.; et al. Adiposity and cardiovascular outcomes in three-year-old children of participants in UPBEAT, an RCT of a complex intervention in pregnant women with obesity. Pediatr. Obes. 2021, 16, e12725. [CrossRef]

34. Patel, N.; Godfrey, K.M.; Pasupathy, D.; Levin, J.; Flynn, A.C.; Hayes, L.; Briley, A.L.; Bell, R.; Lawlor, D.A.; Oteng-Ntim, E.; et al. Infant adiposity following a randomised controlled trial of a behavioural intervention in obese pregnancy. Int. J. Obes. 2017, 41, 1018-1026. [CrossRef]

35. Horan, M.K.; McGowan, C.A.; Gibney, E.R.; Donnelly, J.M.; McAuliffe, F.M. Maternal diet and weight at 3 months postpartum following a pregnancy intervention with a low glycaemic index diet: Results from the ROLO randomised control trial. Nutrients 2014, 6, 2946-2955. [CrossRef]

36. Moran, L.J.; Flynn, A.C.; Louise, J.; Deussen, A.R.; Dodd, J.M. The effect of a lifestyle intervention on pregnancy and postpartum dietary patterns determined by factor analysis. Obesity 2017, 25, 1022-1032. [CrossRef]

37. Skouteris, H.; Hartley-Clark, L.; McCabe, M.; Milgrom, J.; Kent, B.; Herring, S.J.; Gale, J. Preventing excessive gestational weight gain: A systematic review of interventions. Obes. Rev. 2010, 11, 757-768. [CrossRef]

38. Rauh, K.; Kunath, J.; Rosenfeld, E.; Kick, L.; Ulm, K.; Hauner, H. Healthy living in pregnancy: A cluster-randomized controlled trial to prevent excessive gestational weight gain-Rationale and design of the GeliS study. BMC Pregnancy Childbirth 2014, 14, 119. [CrossRef]

39. Kunath, J.; Günther, J.; Rauh, K.; Hoffmann, J.; Stecher, L.; Rosenfeld, E.; Kick, L.; Ulm, K.; Hauner, H. Effects of a lifestyle intervention during pregnancy to prevent excessive gestational weight gain in routine care-The cluster-randomised GeliS trial. BMC Med. 2019, 17, 5. [CrossRef]

40. Günther, J.; Hoffmann, J.; Spies, M.; Meyer, D.; Kunath, J.; Stecher, L.; Rosenfeld, E.; Kick, L.; Rauh, K.; Hauner, H. Associations between the Prenatal Diet and Neonatal Outcomes-A Secondary Analysis of the Cluster-Randomised GeliS Trial. Nutrients 2019, 11, 1889. [CrossRef] [PubMed]

41. Hoffmann, J.; Günther, J.; Geyer, K.; Stecher, L.; Rauh, K.; Kunath, J.; Meyer, D.; Sitzberger, C.; Spies, M.; Rosenfeld, E.; et al. Effects of a lifestyle intervention in routine care on prenatal physical activity-Findings from the cluster-randomised GeliS trial. BMC Pregnancy Childbirth 2019, 19, 414. [CrossRef]

42. Günther, J.; Hoffmann, J.; Kunath, J.; Spies, M.; Meyer, D.; Stecher, L.; Rosenfeld, E.; Kick, L.; Rauh, K.; Hauner, H. Effects of a Lifestyle Intervention in Routine Care on Prenatal Dietary Behavior-Findings from the Cluster-Randomized GeliS Trial. J. Clin. Med. 2019, 8, 960. [CrossRef]

43. Hoffmann, J.; Günther, J.; Stecher, L.; Spies, M.; Geyer, K.; Raab, R.; Meyer, D.; Rauh, K.; Hauner, H. Infant growth during the first year of life following a pregnancy lifestyle intervention in routine care-Findings from the cluster-randomised GeliS trial. Pediatr. Obes. 2021, 16, e12705. [CrossRef]

44. Johar, H.; Hoffmann, J.; Günther, J.; Atasoy, S.; Stecher, L.; Spies, M.; Hauner, H.; Ladwig, K.-H. Evaluation of antenatal risk factors for postpartum depression: A secondary cohort analysis of the cluster-randomised GeliS trial. BMC Med. 2020, 18, 227. [CrossRef]

45. Hoffmann, J.; Günther, J.; Geyer, K.; Stecher, L.; Kunath, J.; Meyer, D.; Spies, M.; Rosenfeld, E.; Kick, L.; Rauh, K.; et al. Associations between Prenatal Physical Activity and Neonatal and Obstetric Outcomes-A Secondary Analysis of the Cluster-Randomized GeliS Trial. J. Clin. Med. 2019, 8, 1735. [CrossRef] [PubMed]

46. Koletzko, B.; Cremer, M.; Flothkötter, M.; Graf, C.; Hauner, H.; Hellmers, C.; Kersting, M.; Krawinkel, M.; Przyrembel, H.; Röbl-Mathieu, M.; et al. Diet and Lifestyle Before and During Pregnancy_Practical Recommendations of the Germany-wide Healthy Start-Young Family Network. Geburtshilfe Frauenheilkd. 2018, 78, 1262-1282. [CrossRef]

47. Haftenberger, M.; Heuer, T.; Heidemann, C.; Kube, F.; Krems, C.; Mensink, G.B.M. Relative validation of a food frequency questionnaire for national health and nutrition monitoring. Nutr. J. 2010, 9, 36. [CrossRef] [PubMed]

48. Kuhn, D.-A. Entwicklung eines Index zur Bewertung der Ernährungsqualität in der Studie zur Gesundheit Erwachsener in Deutschland (DEGS1) German ("Development of a Dietary Quality Index in the German Health Examination Survey for Adults"); Robert Koch Institute: Berlin, Germany, 2017.

49. Chasan-Taber, L.; Schmidt, M.D.; Roberts, D.E.; Hosmer, D.; Markenson, G.; Freedson, P.S. Development and validation of a Pregnancy Physical Activity Questionnaire. Med. Sci. Sports Exerc. 2004, 36, 1750-1760. [CrossRef]

50. Chasan-Taber, L.; Schmidt, M.D. Pregnancy Physical Activity Questionnaire. Can. J. Public Health 2016, 106, e563. [CrossRef] [PubMed] 
51. Ainsworth, B.E.; Haskell, W.L.; Herrmann, S.D.; Meckes, N.; Bassett, D.R.; Tudor-Locke, C.; Greer, J.L.; Vezina, J.; Whitt-Glover, M.C.; Leon, A.S. 2011 Compendium of Physical Activities: A second update of codes and MET values. Med. Sci. Sports Exerc. 2011, 43, 1575-1581. [CrossRef]

52. Rütten, A.; Pfeifer, K. (Eds.) Nationale Empfehlungen für Bewegung und Bewegungsförderung, Edition 1.2.06.17; Bundeszentrale für Gesundheitliche Aufklärung (BZgA): Köln, Germany, 2017; ISBN 978-3-946692-30-0.

53. Donner, A.; Klar, N. Design and Analysis of Cluster Randomization Trials in Health Research; Wiley \& Sons: Chichester, UK, 2000; ISBN 0340691530.

54. Montgomery-Downs, H.E.; Insana, S.P.; Clegg-Kraynok, M.M.; Mancini, L.M. Normative longitudinal maternal sleep: The first 4 postpartum months. Am. J. Obstet. Gynecol. 2010, 203, 465.e1-465.e7. [CrossRef]

55. Sanda, B.; Vistad, I.; Sagedal, L.R.; Haakstad, L.A.H.; Lohne-Seiler, H.; Torstveit, M.K. Effect of a prenatal lifestyle intervention on physical activity level in late pregnancy and the first year postpartum. PLoS ONE 2017, 12, e0188102. [CrossRef]

56. Richardsen, K.R.; Mdala, I.; Berntsen, S.; Ommundsen, Y.; Martinsen, E.W.; Sletner, L.; Jenum, A.K. Objectively recorded physical activity in pregnancy and postpartum in a multi-ethnic cohort: Association with access to recreational areas in the neighbourhood. Int. J. Behav. Nutr. Phys. Act. 2016, 13, 78. [CrossRef] [PubMed]

57. Finger, J.D.; Mensink, G.B.; Lange, C.; Manz, K. Gesundheitsfördernde körperliche Aktivität in der Freizeit bei Erwachsenen in Deutschland. J. Health Monit. 2017, 2, 37-44. [CrossRef]

58. Chamberlain, C.; O’Mara-Eves, A.; Porter, J.; Coleman, T.; Perlen, S.M.; Thomas, J.; McKenzie, J.E. Psychosocial interventions for supporting women to stop smoking in pregnancy. Cochrane Database Syst. Rev. 2017, 2, CD001055. [CrossRef] [PubMed]

59. Kuntz, B.; Zeiher, J.; Starker, A.; Prütz, F.; Lampert, T. Rauchen in der Schwangerschaft-Querschnittergebnisse aus KiGGS Welle 2 und Trends. J. Health Monit. 2018, 3, 47-54. [CrossRef]

60. Zeiher, J.; Kuntz, B.; Lange, C. Rauchen bei Erwachsenen in Deutschland. J. Health Monit. 2017, 2, 59-65. [CrossRef]

61. Vinter, C.A.; Jensen, D.M.; Ovesen, P.; Beck-Nielsen, H.; Tanvig, M.; Lamont, R.F.; Jørgensen, J.S. Postpartum weight retention and breastfeeding among obese women from the randomized controlled Lifestyle in Pregnancy (LiP) trial. Acta Obstet. Gynecol. Scand. 2014, 93, 794-801. [CrossRef]

62. Horan, M.K.; McGowan, C.A.; Gibney, E.R.; Byrne, J.; Donnelly, J.M.; McAuliffe, F.M. Maternal Nutrition and Glycaemic Index during Pregnancy Impacts on Offspring Adiposity at 6 Months of Age-Analysis from the ROLO Randomised Controlled Trial. Nutrients 2016, 8, 7. [CrossRef]

63. Lim, S.; Tan, A.; Madden, S.; Hill, B. Health Professionals' and Postpartum Women's Perspectives on Digital Health Interventions for Lifestyle Management in the Postpartum Period: A Systematic Review of Qualitative Studies. Front. Endocrinol. 2019, 10, 767. [CrossRef] 